\title{
LDL-cholesterol lowering effect of a new dietary supplement: an open label, controlled, randomized, cross-over clinical trial in patients with mild-to-moderate hypercholesterolemia
}

\author{
S. Magno ${ }^{1}$, G. Ceccarini ${ }^{1}$, C. Pelosini ${ }^{1}$, R. Jaccheri ${ }^{1}$, J. Vitti ${ }^{1}$, P. Fierabracci ${ }^{1}$, G. Salvetti ${ }^{1}$, G. Airoldi ${ }^{2}$, M. Minale ${ }^{3}$, \\ G. Saponati ${ }^{3}$ and F. Santini ${ }^{1^{*}}$
}

\begin{abstract}
Background: Hypercholesterolemia is a major risk factor for cardiovascular disorders and requires specific intervention through an adequate lifestyle (diet and physical exercise) and, if necessary, an appropriate drug treatment. Lipid-lowering drugs, although generally efficacious, may sometimes cause adverse events. A growing attention has been devoted to the correction of dyslipidemias through the use of dietary supplements. The aim of this study was to assess the lipid-lowering activity and safety of a dietary supplement containing monacolin $\mathrm{K}$, L-arginine, coenzyme Q10 and ascorbic acid, named Argicolina (A), compared to a commercially available product containing monacolin K and coenzyme Q10, Normolip 5 (N).

Methods: This was a single center, controlled, randomized, open-label, cross-over clinical study enrolling 20 Caucasian outpatients aged 18-75 years with serum LDL-C between 130 and $180 \mathrm{mg} / \mathrm{dL}$. Patients assumed two different dietary supplements ( $\mathrm{A}$ and $\mathrm{N}$ ) both containing monacolin $\mathrm{K} 10 \mathrm{mg}$ for 8 weeks each, separated by a 4-week wash-out period. Evaluated parameters were: Total cholesterol (Tot-C), low density lipoprotein cholesterol (LDL-C), high density lipoprotein cholesterol (HDL-C), triglycerides (TG), fasting blood glucose, aspartate aminotransferase, alanine aminotransferase, creatinekinase, gamma-glutamyl-transpeptidase, brachial arterial pressure and heart rate, measured at the start and at the end of each treatment period. Safety was monitored through the study.

Results: $L D L-C$ decreased by $23.3 \%$ during treatment with $N(p<0.0001)$ and by $25.6 \%$ during treatment with A $(p<0.0001)$; the LDL-C mean reduction was 36.4 (95\% Cl: 45,6-27,1) mg/dL during $\mathrm{N}$ treatment and 40.1 (95\% Cl: 49.2-30,9) mg/dL during A treatment. Tot-C decreased significantly $(p<0.0001)$ within each treatment period. HDL-C increase was negligible during A whereas it was significant during N. TG diminished markedly during $A$ and not significantly during $\mathrm{N}$. The difference between treatments was not statistically significant for all variables. No serious or severe adverse events occurred during the study.

Conclusions: Our results confirm the clinically meaningful LDL-C lowering properties of monacolin K. At variance with a supplement already in the market $(\mathrm{N})$, the novel association (A) of monacolin K with L-arginine, coenzime Q10 and ascorbic acid also produces a significant reduction of triglycerides without significant effects on HDL.
\end{abstract}

Trial registration: ClinicalTrials.gov ID: NCT03425630.

Keywords: Hypercholesterolemia, LDL-cholesterol (LDL-C), Triglycerides, Red reast rice, Monacolin K

\footnotetext{
* Correspondence: ferruccio.santini@med.unipi.it

${ }^{1}$ Obesity Center at the Endocrinology Unit, University Hospital of Pisa, Via

Paradisa 2, Pisa, Italy

Full list of author information is available at the end of the article
}

(c) The Author(s). 2018 Open Access This article is distributed under the terms of the Creative Commons Attribution 4.0 International License (http://creativecommons.org/licenses/by/4.0/), which permits unrestricted use, distribution, and reproduction in any medium, provided you give appropriate credit to the original author(s) and the source, provide a link to the Creative Commons license, and indicate if changes were made. The Creative Commons Public Domain Dedication waiver (http://creativecommons.org/publicdomain/zero/1.0/) applies to the data made available in this article, unless otherwise stated. 


\section{Background}

Cardiovascular diseases (CVDs) rank first as the current leading cause of death [1]. Identification, prevention and management of the risk factors for CVDs are therefore a priority in public healthcare programs [2]. Among CVDs risk factors, one of the most important is dyslipidemia, primarily hypercholesterolemia, which may be corrected through an adequate lifestyle (diet and physical exercise) and, if necessary, an appropriate drug treatment [3]. The results achieved with lipid-lowering drugs, however, are not always satisfactory, and untoward effects (mainly myalgia and myopathies) may sometimes emerge that cause patients to discontinue the treatment $[4,5]$. Over the past few years a growing attention has been devoted to the correction of dyslipidemias through the use of dietary supplements, either because some patients may have milder forms of hypercholesterolemia or as an alternative to statins in patients who may have experienced side effects, although the potential adverse effects caused by supplements have not been fully investigated. There is evidence of a relationship between some food constituents and a reduction in CVD incidence [6-8]. Monacolin $\mathrm{K}$ is a substance obtained during rice fermentation by the fungus Monascus purpureus, used for thousands of years in China to produce rice wine. Fermented rice owes its red color (hence the name "red yeast rice") to various pigments produced by the fungus, including monascorubramin and rubropunctamin. In 1979 Akira Endo of the Tokyo University isolated a metabolite produced by the fungus with a strong inhibitory activity toward the enzyme HMG-CoA reductase (3-hydroxy-3-methyl-glutaryl coenzyme A reductase), which mediates the step from hydroxymethylglutaryl coenzyme A to mevalonic acid early in the pathway to the endogenous synthesis of cholesterol [9]. This metabolite was later characterised as a member of the monacolin group and identified as monacolin $\mathrm{K}$, a structural analogue of lovastatin. Several clinical studies have demonstrated a lipid-lowering effect of monacolin $\mathrm{K}$, alone $[10,11]$ or in association with other compounds [12]. Studies have shown a reduction in serum total cholesterol (Tot-C) between 12 and 30\% after the administration of products containing from 3 to $10 \mathrm{mg}$ of monacolin $\mathrm{K}$, for treatments ranging from 4 weeks up to 1 year [11, 13-15]. Some of these trials have reported a lower incidence of myopathies compared to statins [16]. A recent metanalysis on products containing monacolin $\mathrm{K}$ have shown that the expected mean reduction, compared to placebo, was $37.5 \mathrm{mg} / \mathrm{dL}$ (95\%CI, 30.9-43.7; $p<0.00001$ ) for Tot-C and $33.6 \mathrm{mg} / \mathrm{dL} \quad(95 \% \mathrm{CI}, \quad 27.5-39.8 ; p<0.00001)$ for LDL-cholesterol (LDL-C) [17]. A novel dietary supplement named Argicolina (A) containing monacolin K, L-arginine, coenzyme Q10 and ascorbic acid has been developed by Damor Pharmaceuticals (Naples, Italy). L-arginine is an essential aminoacid, a substrate in the synthesis of nitric oxide (NO) catalysed by the NO-synthetase expressed in the vessel endothelium, therefore acting as a vasodilator and an inhibitor of platelet aggregation [18-23]. Experimental work showed that the association of L-arginine with a statin increased NO production by endothelial cells compared to statin alone [24]. Coenzyme Q has antioxidant activity and is widely used in dietary supplements for subjects with raised lipid levels or cardiovascular risk [25]. Ascorbic acid has antioxidant and vasoprotective activity [26, 27]. The diverse and complementary properties of the components of A suggest that this product may be useful in treating subjects with hyperlipidemia or cardiovascular risk. The aim of this study was to assess the lipid-lowering activity and safety of the proposed formulation in patients with mild-moderate hypercholesterolemia, compared to a commercially available dietary supplement containing monacolin K and coenzyme Q10 (Normolip 5: N) (ESI - Albissola Marina, Savona, Italy). The study was conducted according to a randomized cross-over design.

\section{Methods \\ Patients selection}

Between July 2016 and April 2017 eligible patients were recruited among the outpatients attending the Obesity Center of the Endocrinology Unit 1, Cisanello Hospital, Pisa, Italy. Patients aged 18-75 years with serum LDL-C between 130 and $180 \mathrm{mg} / \mathrm{dL}$, not significantly modified by an appropriate dietetic regimen were considered eligible for the study. Exclusion criteria were: pregnancy or breast-feeding; known liver, renal or muscle diseases; serum triglycerides (TG) greater than $350 \mathrm{mg} / \mathrm{dL}$; previous cardiovascular events; concomitant neoplastic or immunodepressive diseases; use of lipid-lowering drugs or dietary supplements within the last 3 weeks; concurrent use of thiazide diuretics, oral contraceptives containing estrogen or progestogen, systemic corticosteroids; use of psycho-active substances, drug or alcohol abuse; neurological or psychiatric diseases that could affect consent validity or impair the patient's adherence to the study protocol. Thirty patients, all Caucasian, were screened. Ten were excluded during the screening process because they did not fulfill all the inclusion criteria (screening failure). Twenty patients were thus randomized, 10 to the $\mathrm{A}>\mathrm{N}$ sequence and 10 to the $\mathrm{N}>\mathrm{A}$ sequence.

\section{Study design}

The study was conducted in a single center according to a controlled, randomized, open-label, cross-over design. Each patient had to assume, in a randomized sequence, both treatments (A, 1 sachet/day; N, 1 capsule/day) for 8 weeks each separated by a 4-week wash-out period. The study plan included the initial screening visit (V1), an entry visit at start of the first treatment period (V2), a visit at the end of the first treatment period (V3, $56 \pm 5$ days after V2), a wash-out period of 4 weeks ( \pm 5 days), a 


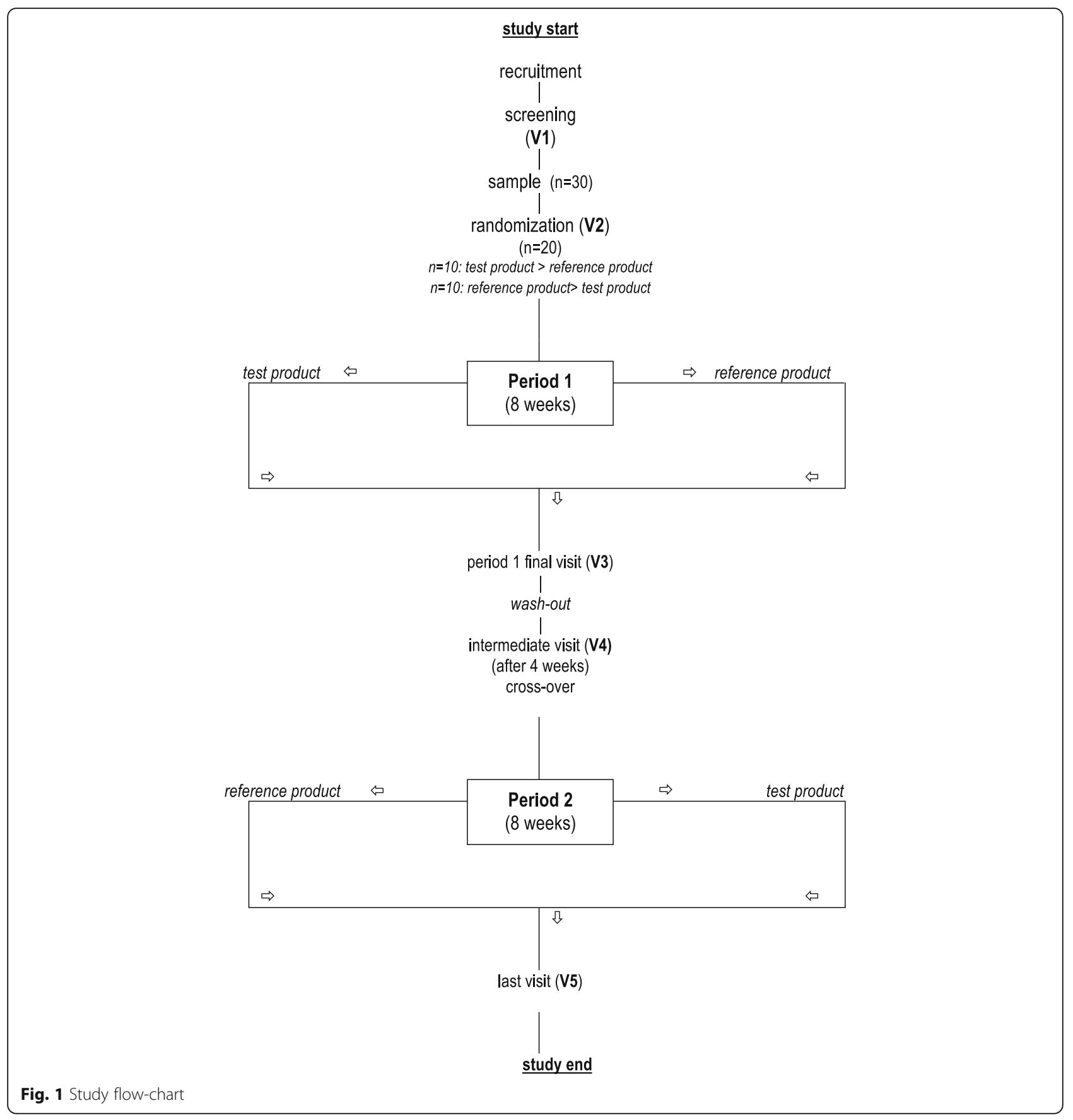

Table 1 Characteristics of the patients at entry

\begin{tabular}{llll}
\hline & all patients (19 patients) & sequence N-A (10 patients) & sequence A-N (9 patients) \\
\hline Gender (F/M) & $13 / 6$ & $7 / 3$ & $6 / 3$ \\
Age (years) & $54.7 \pm 9.3(36-70)$ & $56.2 \pm 9.6(37-69)$ & $53.1 \pm 9.2(36-70)$ \\
Height ( $\mathrm{cm})$ & $168 \pm 11$ & $169 \pm 9$ & $167 \pm 13$ \\
Weight $(\mathrm{Kg})$ & $86 \pm 24$ & $84 \pm 24$ & $87 \pm 26$ \\
Body Mass Index $\left(\mathrm{Kg} / \mathrm{m}^{2}\right)$ & $30.3 \pm 8.2$ & $29.4 \pm 8.2$ & $31.3 \pm 8.6$ \\
\hline
\end{tabular}

Data are expressed as mean \pm SD (min-max range) 
Table $2 \mathrm{LDL}-\mathrm{C}(\mathrm{mg} / \mathrm{dL})$ in the 19 patients who completed the study

\begin{tabular}{llll}
\hline & Argicolina & Normolip & Argicolina - Normolip \\
\hline At start of treatment & $153.1 \pm 11.6$ & $153.1 \pm 14.4$ & $0.0(-7.3+7.3)$ \\
At end of treatment & $113.1 \pm 12.8 *$ & $116.7 \pm 15.4^{*}$ & $-3.7(-9.5+2.1)$ \\
$\Delta$ Final-Initial & $-40.1(-49.2-30.9)$ & $-36.4(-45.6-27.1)$ & $-3.7(-13.0+5.6)$ \\
$\% \Delta$ (Final-Initial) / Initial & $-25.6(-31.1-20.1)$ & $-23.3(-28.7-17.9)$ & $-2.3(-7.4+2.8)$ \\
\hline
\end{tabular}

Data are expressed as mean \pm SD $(95 \% \mathrm{Cl})$, and analyzed by Student's t test for paired data (end vs. start of treatment), ${ }^{*} p<0.0001$

visit at start of the second (crossed over) treatment period (V4), and a visit at the end of the second treatment period (V5, $56 \pm 5$ days after V4) (Fig. 1). Tot-C, LDL-C, HDL-cholesterol (HDL-C), TG, fasting blood glucose, aspartate aminotransferase (AST), alanine aminotransferase (ALT), creatinekinase (CK), gamma-glutamyl-transpeptidase (GGT), brachial arterial pressure and heart rate were measured at V1, V3, V4 and V5. Blood analyses were centrally performed in the laboratory of the Endocrinology Unit using standard enzymatic techniques; LDL-C was directly measured. Clinical safety was monitored throughout the study. If required, the patient could be re-evaluated at any time during the study, aside of the visits scheduled.

\section{Statistical methods}

The minimum level of statistical significance was set to $p<0.05$ two-sided, therefore 95\% confidence limits (95\%CIs) were calculated. All reported $p$-values and CIs are two-sided.

The primary efficacy variable was the LDL-C change between the start and the end of each treatment period, expressed as a percentage of the initial value. Therefore, mean and $95 \% \mathrm{CIs}$ of changes within treatment periods (from V2 to V3 and from V4 to V5) for the experimental and the control treatment, irrespective of sequence, were calculated. The main analysis was the determination of the two-sided $95 \% \mathrm{CI}$ of the between-treatment mean difference in the primary variable, computationally

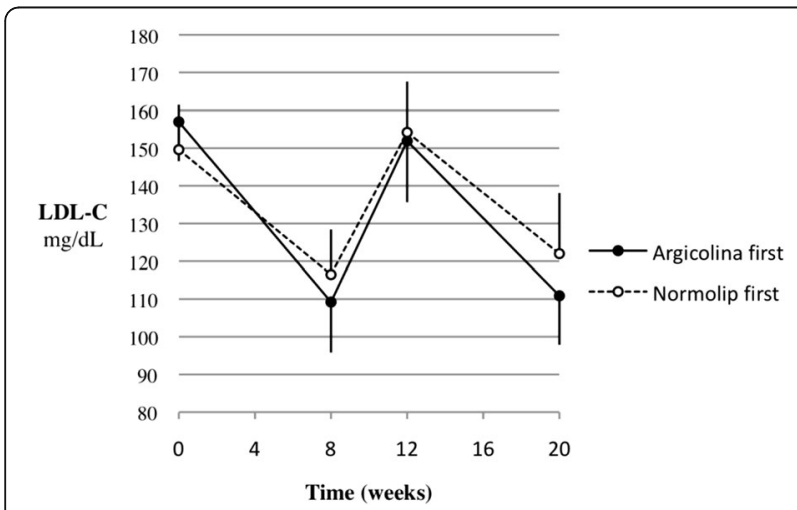

Fig. 2 LDL-C values (mean and SD) through the study, split by treatment sequence analogous to a paired t test. Setting 0.10 (i.e. $10 \%$ of the initial value) as the minimum clinically relevant difference, the two treatments were considered equivalent if the two-sided $95 \% \mathrm{CI}$ of the difference in their LDL-C change from baseline was entirely between -0.10 and +0.10 . Parallel calculations were carried out on absolute, rather than relative to baseline, LDL-C changes. Tot-C and HDL-C were analyzed as described above for LDL-C; for TG levels (which were approximately log-normally distributed) analogous calculations were performed on logarithmic transformations and changes were expressed as ratios. Between-treatment comparisons were expressed as $\mathrm{A}-\mathrm{N}$ differences for cholesterol values and as $\mathrm{A} / \mathrm{N}$ ratios for $\mathrm{TG}$ values. The effects on LDL-C were additionally tested in sensitivity multivariate analyses: variance for cross-over studies on final-baseline changes adjusting for period effects, and analysis of covariance on the difference between the final values adjusted for sequence and for the difference between the

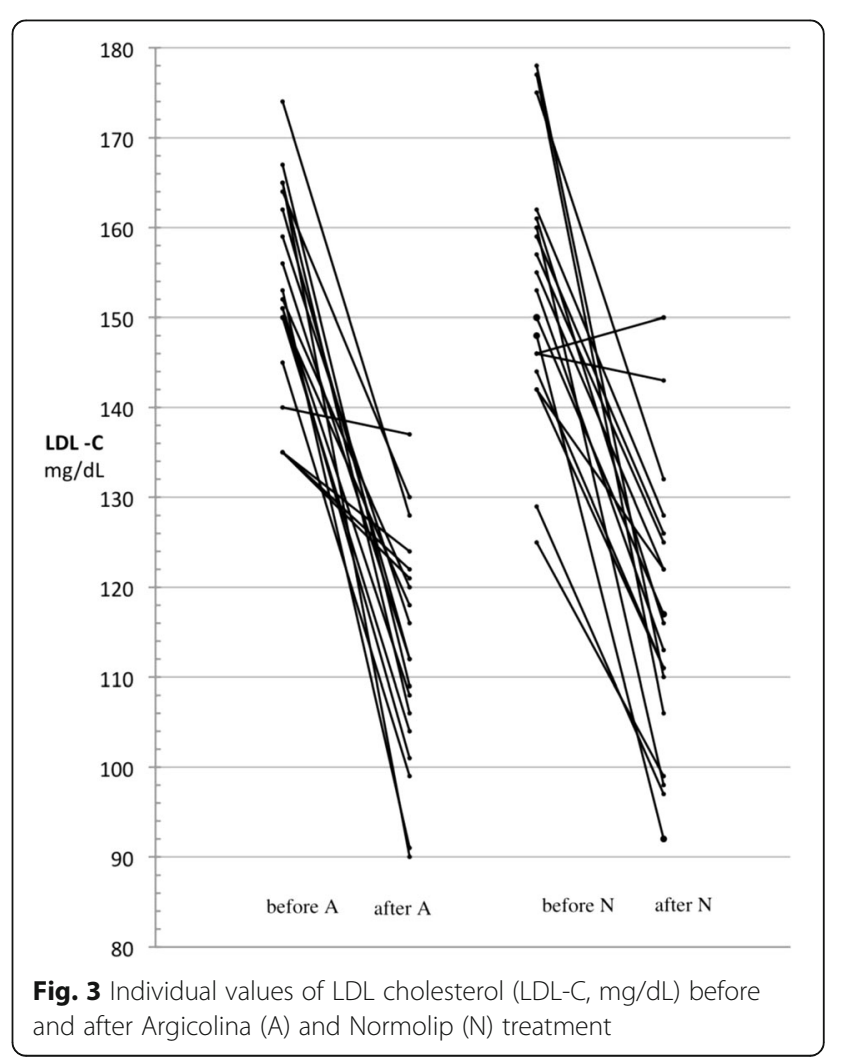


Table 3 Total-C (mg/dL) in the 19 patients who completed the study

\begin{tabular}{llll}
\hline & Argicolina & Normolip & Argicolina-Normolip \\
\hline At start of treatment & $221.1 \pm 16.5$ & $221.6 \pm 18.0$ & $-0.5(-9.8+8.8)$ \\
At end of treatment & $178.3 \pm 16.3^{*}$ & $187.0 \pm 17.1^{*}$ & $-8.7(-15.3-2.1)$ \\
$\Delta$ Final-Initial & $-42.7(-53.6-31.8)$ & $-34.6(-46.1-23.1)$ & $-8.2(-19.9+3.5)$ \\
$\% \Delta$ (Final-Initial) / Initial & $-19.0(-23.5-14.4)$ & $-15.2(-19.8-10.5)$ & $-3.8(-8.2+0.6)$ \\
\hline
\end{tabular}

Data are expressed as mean \pm SD $(95 \% \mathrm{Cl})$, and analyzed by Student's t test for paired data (end vs. start of treatment), ${ }^{*} p<0.0001$

baseline values. Efficacy analyses had to be performed in the intention-to-treat population, i.e. all patients with at least one post-baseline control. A sensitivity analysis of the primary variable was also planned in the per-protocol population, i.e. all patients without major protocol violations. Safety results had to be reported in all patients who had assumed at least one dose of one study drug. Statistical analyses were performed by the Studio Associato Airoldi Cicogna and Ghirri, Milan, using the SAS Software version 9.4 (SAS Inc., Cary, NC).

\section{Sample size}

The sample size was calculated for the main efficacy analysis described above, i.e. the determination of the two-sided $95 \% \mathrm{CI}$ of the between-treatment mean difference in the LDL-C change from baseline. Assuming a standard deviation $(\mathrm{SD})$ of the difference no greater than 0.12 , based on a previous cross-over study for the difference between monacolin $\mathrm{K}$ and placebo [11], it was estimated that 18 patients were required to prove the equivalence with a power of 0.80 . This figure was rounded to 20 enrolled patients allowing for possible exclusions from the analysis.

\section{Results}

\section{Patients characteristics and compliance}

A patient was lost to follow-up after V2 (study entry) and was therefore excluded for analysis. No major violations of the study protocol occurred in the other 19 patients. Therefore the intention-to-treat, per-protocol and safety populations were constituted by the same patients. The demographic characteristics of the 19 patients included in the analysis are shown in Table 1.

The baseline characteristics of the patients assigned to the two different sequences of treatment $(\mathrm{A}>\mathrm{N}$ or $\mathrm{N}>\mathrm{A}$ ) were similar, only slight differences being observed regarding the parameters studied. Ten patients were affected by one or more metabolic or endocrine diseases: obesity $(n=9)$, hypothyroidism treated with L-thyroxine $(n=5)$, and type-2 diabetes mellitus treated with metfor$\min (n=2)$. Six patients were on antihypertensive therapy. The ratio between the number of doses presumably assumed (products delivered minus returned) and the treatment duration in days (equivalent to the number of doses to be taken as per the scheduled one-daily dose regimen), a measure of treatment compliance, was greater than $90 \%$ in all 19 patients who completed the study.

\section{Efficacy}

LDL-C values and their changes during the study are reported in Table 2. LDL-C decreased by $23.3 \%$ during treatment with $\mathrm{N}$ and by $25.6 \%$ during treatment with A. The $\mathrm{A}-\mathrm{N}$ difference, i.e. the mean difference between the within-period changes observed with $\mathrm{A}$ and $\mathrm{N}$, was $-2.3 \%$ (the minus sign meaning a greater reduction during treatment with A compared to N). The $95 \% \mathrm{CI}$ of this difference was between -7.4 and $+2.8 \%$, entirely within the interval of -10 to $+10 \%$ defined as clinical equivalence and used in sample size calculation. In parallel, the absolute (i.e., not referred to the initial value) $\mathrm{LDL}-\mathrm{C}$ mean reduction was $36.4 \mathrm{mg} / \mathrm{dL}$ during $\mathrm{N}$ treatment and $40.1 \mathrm{mg} / \mathrm{dL}$ during $\mathrm{A}$ treatment, the mean A - N difference being $-3.7 \mathrm{mg} / \mathrm{dL}(95 \% \mathrm{CI},-13.0$ to +5.6). LDL-C reductions within each treatment, either as absolute values or expressed as ratio of the initial value, were highly significant $(p<0.0001)$. The results of the analysis of variance adjusting for period effects on final -baseline LDL-C changes were very similar to those of the main analysis above. The mean $\mathrm{A}-\mathrm{N}$ difference relative to baseline was $-2.3 \%(95 \% \mathrm{CI},-7.4$ to $+2.9 ; p=0.36)$, with $p=0.70$ for the period effect. The mean absolute A $-\mathrm{N}$ difference was $-3.8 \mathrm{mg} / \mathrm{dL} \quad(95 \% \mathrm{CI}, \quad-13.4$ to +5.7 ; $p=0.41$ ), with $p=0.52$ for the period effect. The analysis of

Table $4 \mathrm{HDL}-\mathrm{C}(\mathrm{mg} / \mathrm{dL})$ in the 19 patients who completed the study

\begin{tabular}{lll}
\hline & Argicolina & Normolip \\
\hline At start of treatment & $56.3 \pm 15.6$ & $56.6 \pm 16.0$ \\
At end of treatment & $57.7 \pm 15.3$ & $59.2 \pm 17.1^{*}$ \\
$\Delta$ Final-Initial & $+1.4(-0.7+3.5)$ & $+2.6(+0.8+4.3)$ \\
$\% \Delta$ (Final-Initial) / Initial & $+3.0(-1.3+7.3)$ & $+4.3(+1.5+7.1)$ \\
\hline
\end{tabular}

Data are expressed as mean \pm SD, $(95 \% \mathrm{Cl})$, and analyzed by Student's $\mathrm{t}$ test for paired data (end vs. start of treatment), ${ }^{*} p<0.01$ 
Table 5 Triglycerides $(\mathrm{mg} / \mathrm{dL})$ in the 19 patients who completed the study

\begin{tabular}{llll}
\hline & Argicolina & Normolip & Argicolina/Normolip rate \\
\hline At start of treatment & 104.2 & 97.6 & $106.8(96.3118 .4)$ \\
At end of treatment & $86.9^{*}$ & 93.1 & $93.3(81.0107 .4)$ \\
Final / Initial \% & $83.4(72.096 .5)$ & $95.4(85.9106 .0)$ & $87.4(73.3104 .1)$ \\
\hline
\end{tabular}

Data are expressed as geometric mean $(95 \% \mathrm{Cl})$ and analyzed by Student's $t$ test for paired data (end vs. start of treatment) after logarithmic transformation, * $p<0.05$

covariance on the difference between the final values adjusted for sequence and for the difference between the baseline values yielded a mean $\mathrm{A}-\mathrm{N}$ absolute difference of $-3.6 \mathrm{mg} / \mathrm{dL}$, very close to the other estimates reported above, but with a narrower $95 \% \mathrm{CI}(-9.7$ to $+2.5 ; p=0.23)$, with $p=0.49$ for the sequence effect and $p=0.83$ for the difference between the baseline values. No carry-over effect was observed, based on the fact that plasma LDL-C levels at the end of the wash-out were similar to pre-study values (Fig. 2). Individual values at start and end of each treatment showed a decrease of LDL-C in all patients, except for one who changed from 146 to $150 \mathrm{mg} / \mathrm{dL}$ during the first-period treatment with $\mathrm{N}$ (Fig. 3). Irrespective of treatment period, decrease from abnormal $(\geq 130 \mathrm{mg} / \mathrm{dL})$ to normal LDL-C values was obtained in 17 of 19 patients during A treatment (89\%) and in 14 of 17 during $\mathrm{N}$ treatment (82\%; 2 patients were still in the normal range after the wash-out and remained so at the end of treatment). Tot-C, HDL-C and TG values measured before and after each treatment and their differences are reported in Tables 3, 4 and 5 .

Like LDL-C, Tot-C decrease within each treatment period was always highly significant $(p<0.0001)$, while the between-treatment comparison showed a slightly, non-significantly greater reduction with $\mathrm{A}$, both as absolute value $(-8.2 \mathrm{mg} / \mathrm{dL} ; p=0.16)$ and as ratio of baseline $(-3.8 \% ; p=0.086)$.

HDL-C increase was negligible during A and significant during N (Table 4), whereas TG diminished markedly during $\mathrm{A}$ and not significantly during $\mathrm{N}$ (Table 5); the difference between treatments, however, was not statistically significant for both variables $(p>0.30$ and $p=0.12$, respectively). The percent changes of the efficacy variables from start to end of each treatment are summarized in Fig. 4.

\section{Safety}

No serious or severe adverse events occurred during the study; no event required treatment interruption or remedial therapy. Moderate and transient gastrointestinal disturbances occurred in three patients, two receiving $\mathrm{N}$ (constipation and flatulence) and one receiving $\mathrm{A}$ (diarrhea). Other moderate and transient adverse events referred by one patient each were eczema and headache during treatment with $\mathrm{N}$ and myalgia during treatment with A. Serum CK levels above the upper limit of normal (190 U/L) were observed in three patients: one after treatment with A (268 U/L) -previously not treated with $\mathrm{N}$, one after both $\mathrm{A}$ and $\mathrm{N}$ (276 and $246 \mathrm{U} / \mathrm{L}$, respectively), and one after the wash-out period between A and N (320 U/L). No clinically significant change was observed in the serum levels of fasting blood glucose, AST, ALT and GGT, as well as in blood pressure and heart rate measures.

\section{Discussion}

High cholesterol levels are a major risk factor for cardiovascular disorders and may require specific intervention with the prescription of an appropriate drug treatment [3] which has been shown to be able to reduce morbility and mortality in large cohorts of patients [28] and principally based on statins. Red yeast rice, obtained through rice fermentation by the fungus Monascuspurpureus, contains monacolin $\mathrm{K}$ a natural product chemically similar to lovastatin [29]. Monacolin K, similarly to synthetic statins, inhibits the activity of HMG-CoA

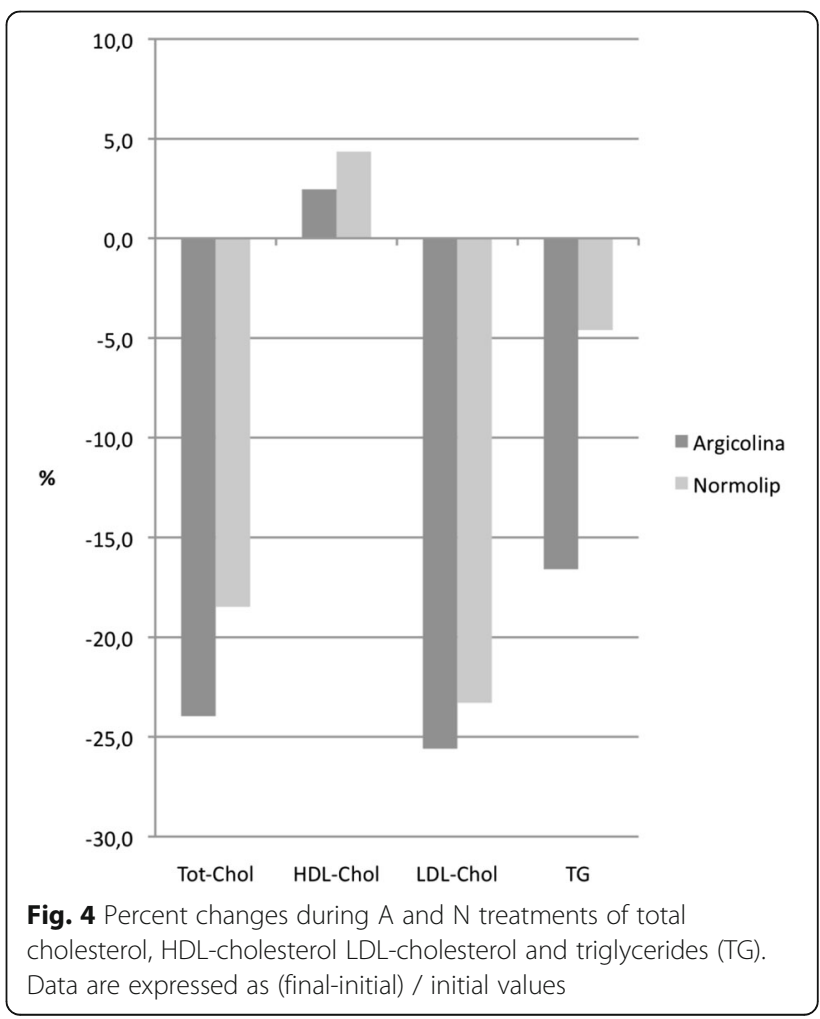


(3-hydroxy-3-methyl-glutaryl coenzyme A) reductase that mediates the production of cholesterol synthesis. In this work we have investigated the lipid lowering capacities of a novel association containing monacolin $\mathrm{K}$, L-arginine, coenzyme Q10 and ascorbic acid. $\mathrm{L}$-arginine is an essential aminoacid with NO-mediated vasodilating properties [18-21], coenzyme $\mathrm{Q}$ has antioxidant activity [25] while ascorbic acid has antioxidant and vasoprotective activity $[26,27]$. The effects of this association were compared with those of a commercially available dietary supplement containing monacolin $\mathrm{K}$ and coenzyme Q10. The study was conducted according to a rigorous randomized cross-over design. Our results were in agreement with previous research on monacolin $\mathrm{K}$ based dietary supplements and showed a statistically significant reduction of LDL-C levels. We were able to document a marked lowering effect of LDL-C (A: $-40.1 \mathrm{mg} / \mathrm{dL}$, $-25,6 \%$; $\mathrm{N}:-36,4 \mathrm{mg} / \mathrm{dL},-23,3 \%$ ), in line with previous reports $[11,13,15,30-32]$, with no difference in efficacy between the two formulations studied. In parallel with the reduction of LDL-C a decrease in Tot-C was documented for both formulations, and HDL-C was increased although reaching significant levels only with $\mathrm{N}$. A significant reduction of triglycerides levels was found exclusively for the novel formulation tested (A). A clinically relevant effect of monacolin K-based supplements on triglycerides has been previously but not consistently reported [10], since this reduction was not documented for $\mathrm{N}$ we speculate that other compounds present in A, probably L-arginine, may have contributed to this effect [33].

Regarding side effects (adverse events) in our study 3/19 patients reported mild and transient gastrointestinal discomfort, and in 3/19 subjects elevation of CK occurred, with no associated myalgia. These figures are similar to what expected and already reported in the literature [34]. Among the limitations of this study we have to mention the open (not blinded) design of the study which was chosen because of the different nature of the pharmaceutical preparations of the compounds tested. The relatively low number of patients enrolled is an additional limitation, the impact of which was attenuated by the crossover design.

\section{Conclusions}

Our results confirm the clinically meaningful lipid lowering properties of monacolin $\mathrm{K}$ and specifically the efficacy of a novel association of it with L-arginine, coenzyme Q10 and ascorbic acid. Furthermore, our results show a good safety profile of this association.

In addition to the very effective LDL-C lowering capacity it is worth noticing the significant reduction of triglycerides level achieved by A, which might be due to the presence of $\mathrm{L}$-arginine in the formulation.

\begin{abstract}
Abbreviations
A: Argicolina; ALT: Alanine aminotransferase; AST: Aspartate aminotransferase; CK: Creatinekinase; CVDs: Cardiovascular diseases; GGT: Gamma-glutamyl-transpeptidase; HDL-C: HDL-cholesterol; HMG-CoA reductase: 3-hydroxy-3-methyl-glutaryl coenzyme A reductase; LDL-C: LDL-Cholesterol; N: Normolip 5; NO: Nitric oxide; TG: Triglycerides; Tot-C: Total cholesterol

\section{Funding}

The study was supported by an unrestricted grant to the Endocrinology Unit (Cisanello Hospital, Pisa, Italy) from Damor Pharmaceuticals. ISPharm srl and Studio Associato Airoldi Cicogna Ghirri were granted from Damor for planning and monitoring the study and to perform the statistical analysis, respectively.
\end{abstract}

\section{Availability of data and materials}

The datasets used and/or analysed during the current study are available from the corresponding author on reasonable request.

\section{Dietary supplements composition}

Argicolina: red yeast rice extract mg 200 (corresponding to monacoline K mg 10), citrinine and aflatoxin-free; L-arginine mg 3500; Coenzyme Q10 mg 50; ascorbate mg 100.

Normolip 5: red yeast rice extract mg 200 (corresponding to monacoline $\mathrm{K}$ mg 10); gamma orizanol mg 90; Coenzyme Q10 mg 10; polycosanols mg 5; chrome picolinate mg 0,2

\section{Authors' contributions}

SM performed the study and was involved in writing the manuscript; GC was involved in writing the manuscript; CP had full access to all the data in the study and took responsibility for the integrity and accuracy; RJ, JV, PF and GS collaborated in recruitment of participants; GA performed the statistical analysis; MM designed the study and wrote the basic manuscript; GS designed the study and was involved in writing the manuscript; FS contributed to the study design, supervised the study and revised the manuscript. All authors read and approved the paper.

\section{Ethics approval and consent to participate}

The study protocol, the patient information sheet, the informed consent form, the letter to general practitioner and the "privacy statement" sheet were examined and approved by the reference Ethic Committee of the investigational study site (Italian National Health Institute. Comitato Etico Area Vasta Nord Ovest [CEAVNO] Toscana, Pisa, Italy) prior to any studyrelated procedure was started. The study initiation was notified to the Italian Ministry of Health. The study was conducted according to the principles defined in the Declaration of Helsinki and amendments, and to the procedures of Good Clinical Practice (whenever applicable), expressed in the guideline set out by the International Conference on Harmonization. The decision on study participation was freely and in written taken by the patient, and it was clarified that the consent could have been withdrawn at any time, without penalty or loss of patient' rights of benefits.

\section{Competing interests}

The authors declare that they have no competing interests.

\section{Publisher's Note}

Springer Nature remains neutral with regard to jurisdictional claims in published maps and institutional affiliations.

\section{Author details}

'Obesity Center at the Endocrinology Unit, University Hospital of Pisa, Via Paradisa 2, Pisa, Italy. ${ }^{2}$ Studio Associato Airoldi Cicogna Ghirri, Via Manzoni 40, Milan, Italy. ${ }^{3}$ ISPharm srl, Via Oberdan 43, Lucca, Italy.

Received: 15 February 2018 Accepted: 10 May 2018

Published online: 24 May 2018

\section{References}

1. WHO Media Centre. Cardiovascular diseases (CVDs). Updated may 2017. http://www.who.int/cardiovascular_diseases/en/. Accessed 17 May 2018. 
2. Piepoli MF, Hoes AW, Agewall S, Albus C, Brotons C, Catapano AL, et al. 2016 European guidelines on cardiovascular disease prevention in clinical practice: the sixth joint task force of the European Society of Cardiology and Other Societies on cardiovascular disease prevention in clinical practice (constituted by representatives of 10 societies and by invited experts) developed with the special contribution of the European Association for Cardiovascular Prevention \& amp; rehabilitation (EACPR). Eur Heart J. 2016; 37:2315-81.

3. Stone NJ, Robinson JG, Lichtestein AH, Bairey Merz CN, Blum CB, Eckel RH, et al. ACC/AHA guideline on the treatment of blood cholesterol to reduce atherosclerotic cardiovascular risk in adults. A report of the American College of Cardiology/American heart association task force on practice guidelines. J Am Coll Cardiol. 2014;63:2889-934.

4. Needham M, Mastaglia FL. Statin myotoxicity: a review of genetic susceptibility factors. Neuromuscolar Disord. 2014;24:4-15.

5. Sirtori CR, Mombelli G, Triolo M, Laaksonen R. Clinical response to statins: mechanism (s) of variable activity and adverse effects. Ann Med. 2012;44: 419-33.

6. Engelfriet P, Hoekstra J, Hoogenveen R, Büchner F, Van Rossum C, Verschuren M. Food and vessels: the importance of a healthy diet to prevent cardiovascular disease. Eur J Cardiovasc Prev Rehabil. 2010;17:50-5.

7. Catalgol B, Batirel S, Taga Y, Ozer NK. Resveratrol: French paradox revisited. Front Pharmacol. 2012;3:141.

8. Volpe R, Sotis G. Nutraceuticals: definition and epidemiological rationale for their use in clinical practice. High Blood Pressure Cardiovasc Prev. 2015;22: 199-201.

9. Endo A. A gift from nature: the birth of the statins. Nat Med. 2008;14: 1050-2.

10. Yang CW, Mousa SA. The effect of red yeast rice (Monascus Purpureus) in dyslipidemia and others disorders. Complement Ther Med. 2012;20:466-74.

11. Cicero AF, Derosa G, Parini A, Maffioli P, D'Addato S, Reggi A, et al. Red yeast rice improves lipid pattern, high-sensitivity $C$-reactive protein and vascular remodelling parameters in moderately hypercholesterolemic italian subjects. Nutr Res. 2013;33:622-8.

12. Solà R, Valls RM, Puzo J, Calabuig JR, Brea A, Pedret A, et al. Effects of poly-byoactive compounds on lipid profile and body weight in a moderately hypercholesterolemic population with low cardiovascular disease risk: a multicenter randomized trial. PLoS One. 2014:9(8):e101978.

13. Ruscica M, Gamaraschi M, Mombelli G, Macchi C, Bosisio R, Pazzucconi F, et al. Nutraceutical approach to moderate cardiometabolic risk: results of a randomized, double-blind and crossover study with Armolipid plus. J Clin Lipidol. 2014;8:61-8.

14. Marazzi G, Cacciotti L, Pelliccia F, laia L, Volterrani M, Caminiti G, et al. Long-term effects of nutraceuticals (berberine, red yeast rice, policosanol) in elderly hypercholesterolemic patients. Adv Ther. 2011;28:1105-13.

15. Gonnelli S, Caffarelli C, Stolakis K, Cuda C, Giordano N, Nuti R. Efficacy and tolerability of a nutraceutical combination (red yeast Rice, policosanols, and Berberine) in patients with low-moderate risk hypercholesterolemia: a double-blind, placebo-controlled study. Curr Ther Res Clin Exp. 2014;77:1-6.

16. Becker DJ, Gordon RY, Halbert SC, French B, Morris PB, Rader DJ. Red yeast rice for dyslipidemia in statin-intolerant patients: a randomized trial. Ann Intern Med. 2009;150:830-9.

17. Li Y, Jiang L, Jia Z, Xin W, Yang S, Yang Q, Wang L. A meta-analysis of red yeast rice: an effective and relatively safe alternative approach for dyslipidemia. PLoS One. 2014;9(6):e98611.

18. Vallance $\mathrm{P}$, Collier J, Moncada S. Nitric oxide synthesised from L-arginine mediates endothelium dependent dilatation in human veins in vivo. Cardiovasc Res. 1989;23:1053-7.

19. Creager MA, Gallagher SJ, Girerd XJ, Coleman SM, Dzau VJ, Cooke JP. L-arginine improves endothelium-dependent vasodilatation in hypercholesterolemic humans. J Clin Invest. 1992;90:1248-53.

20. Böger RH, Bode-Böger SM, Frölich JC. The L-arginine-nitric oxide pathway: role in the atherosclerosis and therapeutic implications. Atherosclerosis. 1996;127:1-11.

21. Bai $Y$, Sun $L$, Yang $T$, Sun $K$, Chen J, Hui R. Increase in fasting vascular endothelial function after short-term oral $\mathrm{L}$-arginine is effective when baseline flow-mediated dilatation is low: a meta-analysis of randomized controlled trials. Am J Clin Nutr. 2009:89:77-84.

22. Tousoulis D, Böger RH, Antoniades C, Siasos G, Stefanadi E, Stefanadis C. Mechanisms of disease: L-arginine in coronary atherosclerosis-a clinical perspective. Nat Clin Pract Cardiovasc Med. 2007;4:274-83.
23. Cooke JP, Tsao PS. Arginine: a new therapy for atherosclerosis? Circulation. 1997:95:311-2.

24. Berthe MC, Bernard M, Rasmusen C, Darquy S, Cynober L, Couderc R. Arginine or citrulline associated with a statin stimulates nitric oxide production in bovine aortic endothelial cells. Eur J Pharmacol. 2011;670: $566-70$.

25. Flowers N, Hartley L, Todkill D, Stranges S, Rees K. Co-enzyme Q10 supplementation for the primary prevention of cardiovascular disease. Cochrane Database Syst Rev. 2014;12:CD 010405.

26. Duarte TL, Lunec J. Review: when is an antioxidant not a antioxidant? A review of novel actions and reactions of vitamin C. Free Radic Res. 2005;39: $671-86$.

27. Aptekmann NP, Cesar TB. Long-term orange juice consumption is associated with low LDL-cholesterol and apolipoprotein B in normal and moderately hypercholesterolemic subjects. Lipids Health Dis. 2013:12:119.

28. Baigent C, Keech A, Kearney PM, Blackwell L, Buck G, Pollicino C, et al. Efficacy and safety of cholesterol-lowering treatment: prospective meta-analysis of data from 90,056 participants in 14 randomised trials of statins. Lancet. 2005:366:1267-78.

29. Nguyen T, Karl M, Santini A. Red yeast rice. Foods. 2017;6(3):E19. https://doi. org/10.3390/foods6030019.

30. Cicero AF, Derosa G, Pisciotta L, Barbagallo C, SISA-PUFACOL Study Group. Testing the short-term efficacy of a lipid-lowering nutraceutical in the setting of clinical practice: a multicenter study. J Med Food. 2015;18:1270-3.

31. Lu Z, Kou W, Du B, Wu Y, Zhao S, Brusco OA, et al. Effect of Xuezhikang, an extract from red yeast chinese rice, on coronary events in a chinese population with previous myocardial infarction. Am J Cardiol. 2008;101: 1689-93.

32. Lin CC, Li TC, Lai MM. Efficacy and safety of Monascus purpureus went rice in subjects with hyperlipidemia. Eur J Endocrinol. 2005;153:679-86.

33. Schulze F, Glos S, Petruschka D, Altenburg C, Maas R, Benndorf R, et al. $\mathrm{L}$-arginine enhances the triglyceride-lowering effect of simvastatin in patients with elevated plasma triglycerides. Nutr Res. 2009;29:291-7.

34. Barrios V, Escobar C, Cicero AF, Burke D, Fasching P, Banach M, Bruckert E. A nutraceutical approach (Armolipid plus) to reduce total and LDL cholesterol in individuals with mild to moderate dyslipidemia: review of the clinical evidence. Atheroscler Suppl. 2017:24:1-15.

\section{Ready to submit your research? Choose BMC and benefit from:}

- fast, convenient online submission

- thorough peer review by experienced researchers in your field

- rapid publication on acceptance

- support for research data, including large and complex data types

- gold Open Access which fosters wider collaboration and increased citations

- maximum visibility for your research: over $100 \mathrm{M}$ website views per year

At BMC, research is always in progress.

Learn more biomedcentral.com/submissions 